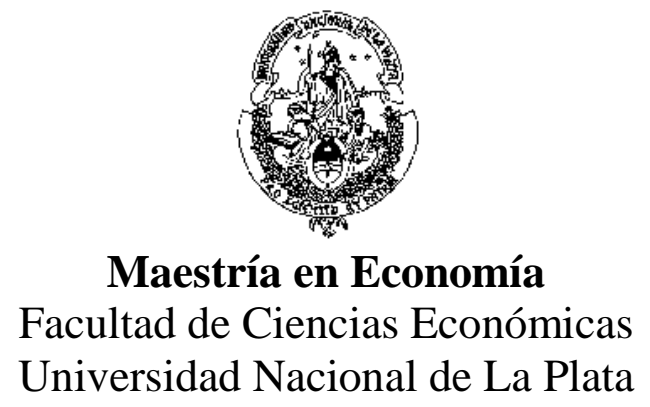

TESIS DE MAESTRIA

ALUMNO

Pablo Lopez Zadicoff

TITULO

Polarizacion en el Consumo de (Ciertos) Bienes de Lujo

DIRECTOR

Jorge Paz

FECHA DE DEFENSA

$12 / 4 / 2009$ 
Universidad Nacional de La Plata

Magister en Economía

\title{
Polarización en el Consumo de (ciertos) Bienes de Lujo
}

\author{
20 de Noviembre de 2009
}

\section{Alumno: Pablo López Zadicoff}

Tutor: Jorge A. Paz

[JEL Codes: D11 - Z13]

\section{Resumen}

El trabajo explora la posibilidad teórica de que la demanda de ciertos bienes presente una curva de Engel en forma de S. Se desarrolla un modelo donde la utilidad depende de dos funcionalidades, así como del nivel de estatus. Se plantea que existen tres bienes, uno de los cuales posee aplicación dual en términos de estatus y funcionalidad. Asimismo los agentes poseen un consumo autónomo de estatus el cuál es función de su dotación de riqueza. Bajo el modelo planteado, se exploran las condiciones que esta función de transformación riqueza-estatus debe satisfacer para originar demandas con comportamiento no monótono ante variaciones de ingreso. Asimismo se postula a dos grupos de bienes (teléfonos celulares y calzado deportivo) como candidatos a exhibir el comportamiento analizado, y se contrasta la hipótesis con datos de consumo para Argentina. 


\section{Polarización en el Consumo de (ciertos) Bienes de Lujo}

\section{A. Introducción}

El presente trabajo se centra en una de las primeras preguntas que todo economista intenta resolver: ¿Por qué demandamos un bien? La definición básica de un libro de texto de Economía es: "Porque nos brinda más utilidad que cualquier alternativa de consumo (o ahorro) alcanzable." Detrás de esta definición, en apariencia sencilla, se esconde la ambigüedad de un término: utilidad.

Para explicar su alcance, tome por ejemplo 2 tiendas de ropa que venden pantalones. Una con un gran anuncio mostrando a sugerentes Adonis y Venus, mientras que la otra muestra un sucio cartel con un nombre más descriptivo que poético. Ambos venden lo mismo (pantalones) y muchas veces las variaciones calidad y diseño son imperceptibles. Sin embargo, los precios de sus productos difieren sustancialmente. La respuesta es que la demanda mira mucho mas allá de lo evidente, verdad que los teóricos del marketing han sabido capitalizar.

Rosen (1974) introdujo en su teoría de precios hedónicos el marco conceptual para resolver el dilema. Según el autor, un bien de consumo en realidad es en si mismo una canasta indivisible de diversos bienes de consumo que son los que otorgan utilidad al demandante. Es decir que en el caso de los pantalones, existen atributos intangibles adicionales a la calidad y el corte, que fundamentan el diferencial de precios.

Las raíces de este valor subjetivo son múltiples y comprenden, siguiendo a Levy (1959), el reflejo de estatus socio-económico (o la fantasía de alcanzarlo), la autoestima, el deseo sexual, y hasta la baja en los costos de transacción. Básicamente, es posible clasificar los orígenes del valor "subjetivo" en dos ramas: los que solucionan problemas de información para el agente (por ejemplo una marca reconocida soluciona problemas de información en cuanto a la calidad del producto) y los que le otorgan un posicionamiento social. El presente trabajo se centrará en el segundo tipo.

El sociólogo y economista Theodor Veblen (1899) con su "Teoría de la clase ociosa" es quizás el precursor de la incorporación de variables subjetivas a la teoría de demanda. El autor comienza su análisis por las clases sociales altas (a la que denomina la clase ociosa) y postula que la demanda de bienes (inclusive la demanda de tiempo de ocio) posee un gran componente (ex ante) secundario de consumo por "emulación pecuniaria". Es su visión que "para ganar y retener la estima de los hombres no basta con poseer riqueza y poder. La riqueza y el poder deben ser puestos en evidencia, pues la estima es solo ganada en evidencia." Veblen denomina a este tipo de consumo como conspicuos consumption y aquí lo denominaremos "ostentación".

Veblen realiza dos observaciones respecto a este fenómeno. La primera, ya anticipada en un trabajo anterior de 1894, es que la necesidad de reconocimiento alcanza a todos los estratos sociales. Como ejemplo el autor menciona al comportamiento de las mujeres y su cuidado por las apariencias. Según Veblen, el poder mantener económicamente a una mujer y comprarle vestimenta elaborada, maquillaje, etc., es un símbolo directo del poder y riqueza de la familia. Es así que si bien las clases trabajadoras no pueden permitirse el ocio de todo el grupo familiar como constante, utilizan a la mujer como una señal de su poder y riqueza. La merma en el consumo de ostentación a medida descendemos la escala socioeconómica es evidente, pero no absoluta. Veblen concluye entonces, "que ninguna sociedad, incluso la más pobre, elimina todo vestigio de ostentación."

En segundo lugar, según el autor, el avance de la industrialización y la sociedad capitalista, fomentan la ostentación por consumo, en lugar de por tiempo de ocio. Por contraposición a una organización social pre-industrial en la que la gente mayormente se conoce; la sociedad capitalista por su anonimato implica una mayor preponderancia del consumo como factor de 
ostentación, lo cual reduce la capacidad de discernimiento entre los distintos estratos sociales. La necesidad de demostración de poder y riqueza debe ser inmediata.

Interpretando la visión de Veblen, podría aseverarse que en la vida capitalista el costo de la ostentación ha disminuido. Esto se debe a que puede prescindirse de la ostentación continua dado que la canasta de consumo promedio del agente no es visible. Por el contrario, el anonimato implica que la gente realiza un juicio "spot" del poder y riqueza de cada agente en base a la información incompleta proveniente de cada avistamiento. Por lo que emular un comportamiento social no alcanzable mediante el consumo de ciertos bienes icónicos, algo que es imposible con información perfecta, es factible en el mundo moderno de información imperfecta. En términos económicos, en la sociedad capitalista moderna la ostentación posee menor costo y mayor retorno. 


\section{B. Revisión Bibliográfica}

Los bienes disponibles en el mercado se caracterizan en inferiores, normales o superiores dependiendo de si la elasticidad ingreso de su demanda es negativa, positiva y menor a uno o superior a la unidad respectivamente. La intención del presente trabajo es explorar modelos que permitan concluir que un bien posee elasticidades ingreso - y una curva de Ángel asociada - con signo cambiante a medida que va cambiando la restricción presupuestaria de los agentes.

Esto se origina, siguiendo a Rosen (1974) en el hecho de que cada bien comerciado en el mercado es en realidad un conjunto indivisible de cualidades, y que son en realidad estas últimas las que dan utilidad al agente. Es decir que la demanda de bienes transados en el mercado es en realidad una demanda derivada por las características de los mismos. Este espectro de $n$ dimensiones puede agruparse en dos: las características funcionales objetivas y las subjetivas, entre ellas la capacidad de dar una imagen de posición social (estatus) al consumidor.

El estatus es un fenómeno social, por lo que para que el consumo de cierto bien tenga algún impacto sobre el estatus, el mismo debe ser observado por la sociedad. Es evidente que el nivel de ingreso es una fuente indiscutible de estatus social; pero el mismo es a priori invisible para la sociedad. Sin embargo, a mayor nivel de ingresos las personas acceden a una mayor cantidad de consumo y a una mejor calidad del mismo, factor que si es percibido.

Lógicamente, no todos los bienes poseen capacidad de dar estatus al consumidor. Por ejemplo, el consumo de almohadas, o de cepillos de dientes, posee una muy baja (sino nula) visibilidad; por lo que es poco factible que aporten algún elemento de estatus. En el otro extremo se encuentran los bienes de uso diario, como ser un teléfono celular, artículos de vestimenta o una salida al cine al teatro o a cenar que; entre otros, son observados en mayor o menor medida por la sociedad. ${ }^{1}$

En otras palabras, uno puede considerar que cada bien posee estas dos facetas: una funcional y otra representativa. Ahora bien, no necesariamente la faceta representativa es explícita, sino que muchas veces el poder acceder a un bien "objetivamente" mejor o a mayor abundancia de bienes refleja el estatus de la persona.

Si consideramos que el estatus es un argumento en la función de utilidad de los agentes con rendimientos positivos pero decrecientes a escala, es de esperar que la demanda de estatus por un agente se incremente vis-a-vis el incremento en su ingreso. Sin embargo, es su mismo ingreso el que de algún modo le fija un consumo mínimo de status. Por lo tanto, las personas con bajo nivel de ingreso probablemente posean al momento de enfrentar su decisión de consumo, una utilidad marginal del estatus mayor que las personas de mayor ingreso. Esto se debe a que el consumo de estatus "autónomo" de los agentes de cierto nivel (medio) de ingresos estará por encima de su nivel de consumo en el óptimo. Por lo tanto, ellos no tendrán incentivos a salir a "comprar" estatus al mercado, tal como lo hacen los sectores bajos o altos.

Asumiendo que existen bienes con alta capacidad de generar estatus, es probable que los sectores de bajos recursos sean más proclives a adquirirlos que ciertos sectores con mayores recursos. Por lo tanto, lo que se plantea es que existen bienes los cuales se comportan como bienes normales o bienes inferiores, dependiendo del tramo de ingreso que se este analizando.

\footnotetext{
1 Un tercer grupo, es el de bienes que poseen primariamente una finalidad representativa, como ser las alhajas. Por lo general la literatura se ha ocupado de este tipo de bienes como el principal mercado "implícito" de estatus. El presente trabajo, por el contrario, se ocupa de bienes de estatus que poseen una valoración "funcional" no despreciable.
} 
Liebenstein (1950) identifica 3 factores extrínsecos a la funcionalidad de un bien que afectan su demanda. El los llama el efecto manada, el efecto snob, y el efecto Veblen. El primero se basa en que el consumo del bien otorga un sentido de pertenencia al club de consumidores del mismo, por lo que a más consumidores mayor es el bienestar derivado de pertenecer a ese club. Por el contrario, el efecto snob se origina en el hecho de que el consumo de un bien puede definir a una persona, y la capacidad de definición varia inversamente con la cantidad de consumidores. Por último, el efecto Veblen se basa en la señal de poder que brinda el objeto consumido, la cuál es mayor cuanto mayor sea su precio.

En el presente trabajo, no se indagan las razones por las que un bien posee una valoración social positiva, sino que simplemente se los toma como dados. ${ }^{2}$ Sin embargo, al igual que lo que sucede con el efecto manada, el bien objeto de análisis otorga un sentido de pertenencia a cierto club (de estatus), pero no se analiza si el beneficio de pertenecer a dicho club no esta sujeto a externalidades de red. Por el contrario, según el motivo snob cuanto más exclusivo el bien (cuyo valor principal es el de generar un mercado para el estatus), mejor su capacidad como señal. El presente trabajo por su parte, tampoco indaga en esta problemática.

Asimismo, los bienes analizados se diferencian de los bienes tipo Veblen (similares a los Giffen pero con otra fundamentación) ya que aquí las curvas de demanda de los mismos continúan teniendo pendiente negativa.

En cuanto a otras teorías existentes sobre la demanda de bienes de estatus por los sectores bajos de la sociedad, se destacan las teorías de "clubes." Estas se basan en la idea de que cada agente se asocia dentro de un grupo de pertenencia, presumiblemente de su clase social, y por lo tanto trata de distinguirse dentro de su propio club mediante la adquisición de bienes de estatus. Es decir, que en los modelos de clubes un agente no intenta ganarse el reconocimiento de toda la sociedad sino de su grupo de pertenencia. ${ }^{3}$ Las teorías de clubes predicen una demanda de bienes de ostentación por todas las clases sociales, pero monótonamente creciente a medida que aumentamos el poder adquisitivo de cada club. Por el contrario, la teoría aquí expuesta intenta explicar patrones de consumo de ciertos bienes de ostentación no uniformes. ${ }^{4}$

Otra rama de la literatura relacionada es encabezada por Ireland (1994), quien incorpora la utilidad del estatus como un componente aditivo separable a la función de utilidad. Al igual que en el modelo planteado, el estatus depende del consumo del bien visible (Z), directa e indirectamente. El vínculo indirecto se da a través de una función $\mathrm{g}(\mathrm{z})$ que es la percepción endógena de la sociedad del consumo del bien no visible en base al consumo visible. La virtud de esta formulación es que $\mathrm{g}(\mathrm{z})$ es definida por la sociedad de forma de lograr un equilibrio separador entre los agentes. A diferencia de Ireland (1994), el presente trabajo considera que existe un reflejo "autónomo" de estatus por cada agente.

\footnotetext{
2 Para un caso contrario, ver Frank (1985).

${ }^{3}$ Ver por ejemplo McBride (2001), quien utiliza grupos etarios, Luttmen (2005) o Perez Truglia (2007) quienes toman un enfoque "geográfico" o Van de Stad et. Al (1985) quienes aseveran que la utilidad es función del ranking percibido por el agente en su distribución del ingreso de referencia. Notar que igualmente la definición de clubes puede ser bastante amplia, Por ejemplo Easterlin (1995) toma a todos los habitantes de un país como el club de referencia, lo cual implica un comparable grado de amplitud con el modelo aquí presentado.

${ }^{4}$ La reconciliación de teorías, podría darse si consideramos que la presente se refiere a bienes particulares mientras que la de clubes corresponde a bienes genéricos de estatus. De ser asi, cada clase social tomará un grupo de bienes particular como símbolo de estatus, cuyo costo absoluto será creciente vis-a-vis el ingreso. Sin embargo, dado que los bienes tomados como referencia son distintos, una clase social no tendrá motivos de estatus para demandar los bienes "señal" de la clase anterior, por lo que puede ser que su consumo baje (ya que solo tendrá motivos funcionales para demandar). Es decir, que la demanda global de bienes de estatus será creciente con el ingreso, pero dado que los bienes de ostentación son no uniformes entre clases, cada uno de estos puede mostrar comportamientos no uniformes en su curva de Engel.
} 
Por último, hay una rama (mayormente) empírica de la literatura de estatus - Ej., Charles et. al (2007), Lamont et. al (2001) - que miden el consumo diferenciado por grupos raciales de ciertos bienes suntuarios. Este fenómeno puede ser fácilmente incorporado a la presente formulación, si se supone que cada grupo racial posee (en la formulación presentada) una función de estatus autónomo específica. ${ }^{5}$

A continuación se desarrollará un modelo teórico compatible con la proposición de existencia de una curva de Engel para un bien con signo no uniforme, basada en un modelo con un componente de estatus autónomo vinculado a la dotación de los agentes, y a la posibilidad de participar de un mercado implícito de estatus mediante la adquisición de un bien con funcionalidad dual (estatus-consumo).

5 En términos del modelo a desarrollar, se podría decir que $L(M)$ se afectado por otras características demográficas como ser la raza. 


\section{Modelo Teórico}

En esta sección se desarrollará un modelo teórico compatible con la discusión precedente. En una primera instancia se trabajará con una forma funcional indeterminada con la finalidad de obtener conclusiones sobre las condiciones de primer orden del problema de maximización de utilidad de la forma más general posible. Luego, aunque a riesgo de perder algo de generalidad, se tomará una función de tipo Cobb-Douglas para profundizar en el análisis.

\section{C.1 Modelo General}

Primero asumimos que la función de utilidad de los agentes depende del consumo de dos (grupos de) funcionalidades $\left(F_{1}\right.$ y $\left.F_{2}\right)$, así cómo de la percepción de estatus que el agente genera (S). La primer funcionalidad $\left(F_{1}\right)$ comprende el consumo de bienes de baja visibilidad social. Como su consumo no es mayormente observado por el resto de los agentes, su capacidad de generar estatus es muy limitada. La segunda $\left(F_{2}\right)$ se trata por el contrario del consumo de bienes de alta exposición, lo cual lo hace socialmente evidente. En otras palabras, cuando el agente consume bienes de este tipo la sociedad observa integralmente su consumo.

Para satisfacer estos dos grupos de funcionalidades, el agente tiene disponibles en el mercado tres (conjuntos de) bienes. El primero, $X$, impacta exclusivamente sobre el consumo de $F_{1}$. Los restantes, $Y$ y $Z$, hacen lo propio con el consumo de $F_{2}$. Es así que la utilidad de los agentes depende del consumo de dos grupos de funcionalidades, uno con baja visibilidad social y otro con alta. Esas funcionalidades son obtenidas por el agente mediante la compra de 3 (grupos de) bienes con aplicaciones determinadas. ${ }^{6}$

Tal como se mencionó, el tercer argumento de la función de utilidad de los agentes, es el estatus (S). Se asume que $\mathrm{S}$ depende de dos variables. La primera es del ingreso del agente (M), el cual es exógeno; y en segunda instancia de ciertas decisiones de compra. En particular, la compra de Z posee un impacto positivo sobre $S^{7}{ }^{7}$ Es $Z$ el bien sobre el que se centrará el análisis.

En resumen, la compra de $X$ determina por si mismo el consumo de $F_{1}$, mientras que el consumo de $F_{2}$ es determinado en forma conjunta por la compra de $Y$ y $Z{ }^{8}$ El nivel de $S$ alcanzado por el agente posee un mínimo exógeno dado por su dotación de riqueza, y puede ser elevado mediante la compra del bien Z. Por lo tanto la función de utilidad de los agentes queda definida como:

$$
U=U\left[F_{1}(X), F_{2}(Y, Z), S(M, Z)\right]
$$

\footnotetext{
${ }^{6}$ A lo largo del trabajo se hablara en términos de Consumo de los argumentos de la función de utilidad $\left(F_{1}, F_{2}\right.$, $S)$, y compra de bienes $(X, Y, Z)$ que facilitan la obtención de dichas funcionalidades.

7 Si bien es de esperar que todos los bienes tengan cierto impacto sobre S, esto ya fue captado al incluir M como generador exógeno de S. Lo que se supone en realidad es que Z posee un impacto diferencial en $\mathrm{S}$ con respecto al resto de los bienes.

${ }^{8}$ A diferencia de otros trabajos (ver por ejemplo Becker et. al. (2003)) aquí se plantea que el bien de ostentación posee un impacto no despreciable en su funcionalidad "práctica" sino que compite como sustituto directo de otros bienes sin componente de ostentación. Otro ejemplo, es Moffit (1983) quien plantea y prueba empíricamente que algunos agentes están dispuestos a sacrificar ingresos asistenciales (algo con valor funcional nulo) a cambio de no sufrir el estigma de recibir asistencia.
} 
Para la cual se cumple que $U_{i}^{\prime}>0, U_{i i}^{\prime}<0$, y $U^{\prime \prime}{ }_{i j}>0$; para i, j $=F_{1}, F_{2}$, S y para i, j= X, Y, Z.

Por simplicidad se asumen las siguientes relaciones. Una unidad comprada del bien $X$ equivale a una unidad de consumo de $F_{1}$, por lo que $F_{1}=X$. Por su parte se asume que $X$ y $Z$ son sustitutos perfectos para obtener el $F_{2}$, por lo que $F_{2}(Y, Z)=F_{2}(Y+Z)$. Por último, se asume que el precio del bien $Z$ es mayor al del bien $Y$, dado que caso contrario el problema seria trivial. De aquí derivamos

$$
U=U\left[X, F_{2}(Y+Z), S(M, Z)\right]
$$

Nótese que para cada nivel de ingreso exógeno $\mathrm{M}$, existe un mapa de curva de indiferencias nuevo, dado que el mismo depende del estatus que el ingreso genera.

El problema de maximización, en el cual deben incorporarse restricciones de no negatividad para los bienes $X, Z$ es el siguiente:

$\operatorname{Max} L=U\left[X, F_{2}(Y+Z), S(M, Z)\right]+\mu\left(M-p_{x} X-p_{y} y-p_{z} Z\right)+\eta\left(Y-g^{2}\right)+\phi\left(Z-h^{2}\right)$

Las condiciones de primer orden del problema (CPOs) cuando el consumo de todos los bienes es positivo implican que:

$$
\begin{aligned}
& \frac{U_{F 1}^{\prime}}{U_{F 2}^{\prime}}=\frac{P_{X}}{P_{Y}} \\
& \frac{U_{S}^{\prime}}{U_{F 2}^{\prime}}=\frac{P_{Z}-P_{Y}}{P_{Y}} \\
& \frac{U_{S}^{\prime}}{P_{Z}-P_{Y}}=\frac{U_{F 2}^{\prime}}{P_{Y}}=\frac{U_{F 1}^{\prime}}{P_{X}}
\end{aligned}
$$

La condición B.1 refleja la tasa marginal de sustitución entre el nivel deseado de $F_{1}$ y $F_{2}$ equivale a la relación de precios entre el bien $\mathrm{X}$ y el bien $\mathrm{Y}$, que son los bienes que poseen impacto exclusivo sobre el consumo de dichas funcionalidades. Nótese entonces, que la decisión de compra del bien $X$ depende exclusivamente de la decisión de consumo del factor $F_{2}$, pero es independiente de la decisión del mix de compra entre $Y$ y $Z$.

La ecuación B.2 implica que el cociente entre la utilidad marginal del estatus y la de la funcionalidad a la que se aplica el bien que genera estatus $\left(F_{2}\right)$ es equivalente a la prima porcentual del precio del bien que genera estatus $(Z)$ sobre el precio de su sustituto en términos de funcionalidad $(Y)$. La condición B.2 permite pensar en una curva de demanda con pendiente negativa en la curva de demanda del bien $Z$. Cuando el bien $Z$ se hace más caro, la utilidad marginal del estatus crece con respecto a la utilidad marginal de la funcionalidad afectada por dicho bien. Dado que se asume que los 3 factores que afectan la utilidad son "normales", frente a un aumento de precios el consumo de todos debe bajar. Y la única forma de reducir el consumo de $\mathrm{S}$ es mediante una merma en la compra del bien $Z$. Este resultado es contrario a lo que sucede con los bienes de tipo Veblen, los cuales por definición poseen pendiente positiva en su curva de demanda.

Sobre esta condición es también posible inferir una política de precios desde el punto de vista del productor. El precio de un bien de estas características debe ser fijado con una prima equivalente al cociente entre la utilidad marginal del estatus del agente representativo y la utilidad marginal de la funcionalidad a la que este tipo de bien sustituye. 
Por último, la condición B.3 refleja la igualación de utilidades marginales por peso gastado en el punto óptimo. Nótese, que el "precio" de la funcionalidad $F_{2}$ equivale al precio del bien con aplicación exclusiva a la misma, mientras que el "precio" del estatus equivale al diferencial de precios entre los bienes sustitutos para dicha funcionalidad. En algún sentido, esto refleja la idea de Veblen de que los bienes de alta exposición poseen una demanda mayormente explicada por el estatus y no por la funcionalidad.

La existencia de un consumo autónomo de $S$ dada por la dotación exógena $M$, puede originar situaciones en las que el agente desearía comprar cantidades negativas de Z para reducir el consumo de S. Lógicamente esto resulta imposible, por lo que el agente decidirá no comprar Z (solución de esquina). En este caso, es fácil ver que el problema del agente se reduce al modelo tradicional de 2 bienes.

Una segunda solución de esquina puede darse si el agente opta por no comprar Y. Esto en teoría podría darse si un agente posee un nivel de $S$ autónomo muy bajo. En este caso, las las CPOs del modelo implican que:

$$
\frac{U_{F 2}^{\prime}+U^{\prime}{ }_{S}}{P_{Z}}=\frac{U_{F 1}^{\prime}}{P_{X}}
$$

Aquí se repite la (esperable) igualación de utilidad marginal sobre precio en el óptimo, pero aquí el consumo del bien $Z$ aporta utilidad tanto por medio del factor status como por el aporte a la funcionalidad publica (ya que el bien alternativo "Y" no es consumido). En algún sentido se puede interpretar que el fenómeno de estatus "subsidia" al consumo del bien con exposición.

\section{C.2 Modelo Cobb-Douglas Genérico}

A riesgo de perder algo de generalidad, se asume que la función de utilidad toma la siguiente forma:

$$
\left.U=F_{1}(X)^{a} F_{2}(Y+Z)^{b} S L(M)+Z\right)^{(1-a-b)}
$$

Asimismo, se asume que $S(M, Z)=S(L(M)+Z)$, es decir que el bien $Z$ posee rendimientos constantes para generar $S$ mientras que $M$ posee rendimientos variables descriptos por la función $L(M)$. Se remarca que esta simplificación se realiza para mayor claridad expositiva, ya que es posible obtener conclusiones similares otorgando a $Z$ otro perfil de rendimientos a escala en la generación de S; siempre que se respete que $U^{\prime \prime}{ }_{z z}<0 .{ }^{9}$

Primero se buscará la solución cuando la compra de todos los bienes es positiva. Al resolver el problema de maximización (B) introduciendo la función (C) se obtienen las siguientes CPOs:

$$
\frac{a}{b} \frac{Y+Z}{X}=\frac{P_{X}}{P_{Y}}
$$

\footnotetext{
9 Notar que la función (C) cumple con U'zz<0. La prueba se reduce a verificar que $2<1-b /(1-a-b)$ * $[\mathrm{L}(\mathrm{M})+\mathrm{Z}] /(\mathrm{x}+\mathrm{z})+(\mathrm{a}+\mathrm{b}) / \mathrm{b}\{[\mathrm{L}(\mathrm{M})+\mathrm{Z}] /(\mathrm{x}+\mathrm{Z})\}^{\wedge}-1$. Esta prueba es más estricta que la siguiente: $2<[L(M)+Z] /(\mathrm{x}+\mathrm{Z})+$ $\{[L(M)+Z] /(x+z)\}^{\wedge}-1$. Dado que el mínimo de una expresión como la anterior $(x / y+y / x)$ se da cuando $x=y$, su valor mínimo es 2 . Esto implica que la función de utilidad (C) cumple con rendimientos decrecientes a escala para el bien $Z$.
} 


$$
\begin{aligned}
& \frac{1-a-b}{b} \frac{Y+Z}{L(M)+Z}=\frac{P_{Z}-P_{Y}}{P_{Y}} \\
& M=p_{x} X+p_{y} y+p_{z} Z
\end{aligned}
$$

Trabajando con las restricciones se llega a la siguiente solución:

$$
\begin{aligned}
& Z=\frac{1-a-b}{P_{Z}-P_{Y}} M-(a+b) L(M) \\
& Y=\frac{b P_{Z}+a P_{Y}-P_{Y}}{P_{Y}(1-a-b)} Z+\frac{\left(P_{Z}-P_{Y}\right)}{P_{Y}} \frac{b}{1-a-b} L(M) \\
& Y=\frac{b P_{Z}+a P_{Y}-P_{Y}}{P_{Y}\left(P_{Z}-P_{Y}\right)} M+\frac{b P_{Z}+a P_{Y}}{P_{Y}} L(M) \\
& X=\frac{\left(P_{Z}-P_{Y}\right)}{P_{X}} \frac{a}{1-a-b}[L(M)+Z] \\
& X=\frac{a}{P X}\left[M+\left(P_{Z}-P_{Y}\right) L(M)\right]
\end{aligned}
$$

Para que esta sea una solución factible debe darse que:

De D.1, para que $Z>0 \quad \frac{1-a-b}{a+b}>\frac{L(M)}{M}\left(P_{Z}-P_{Y}\right)$

De D.2, para que $Y>0 \quad \frac{P_{Y}-b P_{Z}-a P_{Y}}{b P_{Z}+a P_{Y}}<\frac{L(M)}{M}\left(P_{Z}-P_{Y}\right)$

La condición E.1 es factible de interpretación. Para que la demanda de Z sea positiva es necesario que el valor de mercado del nivel de estatus autónomo (i.e., el lado derecho de la inecuación E.1) sea menor a la elasticidad estatus-utilidad (1-a-b) sobre la suma de la elasticidad de las funcionamientos-utilidad $(a+b)$. La condición E.2 no posee una interpretación directa.

Se remarca que es imposible que ambas condiciones no se satisfagan y que por ende la solución óptima sea $Y=Z=0$. Esto es intuitivamente esperable dado que la función de utilidad (C) valuada en el punto $(X, 0,0)$ otorga utilidad 0 . Formalmente, la prueba es que $\frac{P_{Y}-b P_{Z}-a P_{Y}}{b P_{Z}+a P_{Y}}<\frac{1-a-b}{a+b}$ para todo valor posible de las variables. ${ }^{10}$

De las condiciones precedentes, es posible inferir la importancia del comportamiento de la función $\mathrm{L}(\mathrm{M})$. Nótese que si la misma presenta rendimientos constantes a escala, la demanda por cada uno de los bienes es siempre positiva o siempre nula para cualquier nivel de $\mathrm{M}$.

\footnotetext{
${ }^{10}$ Siempre que Pz > Py esta condición se satisface.
} 
Por el contrario, si la función presenta rendimientos decrecientes a escala, es posible que la demanda de $Z$ sea nula a bajos niveles de $M$ y pase a ser positiva luego de cierto umbral $M^{*}$. Mientras que con la demanda de $Y$ puede ser positiva a bajos niveles de $M$ y nula luego de cierto umbral $\mathrm{M}^{* *}$. Lo inverso sucede si $\mathrm{L}(\mathrm{M})$ presenta rendimientos crecientes a escala.

Es por lo tanto posible concluir que si L(M) presenta rendimientos a escala no uniformes, es posible encontrar que a medida que la dotación de $M$ aumenta las demandas de $Z$ e $Y$ pueden alternar tramos positivos con nulos. ${ }^{11}$ Sin embargo, para completar la derivación de la curva de Engel, es necesario indagar cómo se comportan las demandas de $Z$ e $Y$ cuando son positivas de acuerdo a las demandas resultantes del problema de maximización.

Cuando la demanda de ambos bienes es positiva, la derivada de $Z$ con respecto a $M$ es:

$$
d Z / d M=\frac{1-a-b}{P_{Z}-P_{Y}}-(a+b) L^{\prime}(M)
$$

Nuevamente, el signo de la ecuación depende de la magnitud de L'(M) (no de su signo el cual se asume siempre positivo). Si $L^{\prime}(M)>(1-a-b) /[(a+b)(P z-P y)] d Z / d M$ es negativo, mientras que caso contrario positivo. Notar que la probabilidad de que la derivada sea negativa aumenta cuanto menos importancia tiene el estatus en la función de utilidad, y cuanto más caro sea el precio del estatus $\left(P_{z}-P_{y}\right) \cdot{ }^{12}$

Por lo tanto, si la $L(M)$ presenta rendimientos decrecientes(crecientes) a escala, es factible que en un comienzo la curva de Engel del bien tenga un tramo con pendiente negativa(positiva) hasta cierto umbral en que pase a tener pendiente positiva(negativa).

El bien $\mathrm{Y}$, por su parte presenta la siguiente derivada con respecto a $\mathrm{M}$ :

$$
d Y / d M=\frac{b P_{Z}+a P_{Y}-P_{Y}}{P_{Y}\left(P_{Z}-P_{Y}\right)}+\frac{b P_{Z}+a P_{Y}}{P_{Y}} L^{\prime}(M)
$$

Aquí el signo de la derivada depende tanto de L'(M) como de los parámetros a, b y los precios. Si el signo del numerador del primer término de D.2.2.dm es positivo, la curva de Engel del bien $Y$ tendrá pendiente positiva consistentemente. Si el signo del mismo es negativo, es posible encontrar tramos con pendiente negativa de la curva de Engel del bien $Y$ dependiendo de los valores de los parámetros y de la forma de $L(M)$.

En cuanto a las derivadas con respecto al precio del bien $Z$, se observa que mientras la derivada del bien $Z$ con respecto a su propio precio es negativa, la del bien $Y$ es positiva. ${ }^{13}$ Esto implica, tal como se insinuó en la sección precedente, que el tipo de bienes analizado por la presente función de utilidad no se corresponde con los bienes tipo Veblen en los que la demanda del mismo reacciona positivamente con su precio. Asimismo, la compra del bien sustituto $(Y)$ en términos de funcionalidad posee relación directa con el precio del bien Z.

\footnotetext{
${ }^{11}$ Con la salvedad de que ambas demandas no pueden ser nulas en el mismo nivel de $\mathrm{M}$.

${ }^{12}$ Resulta interesante también ver como varia la participación en el consumo total del bien $\mathrm{Z}$ a medida que varia M. $d(Z / M) / d M=\frac{a-b}{M^{2}}\left(L(M)-L^{\prime}(M) M\right)$. El signo de esta ecuación es negativo cuando D.1.dm es negativo y depende de la forma de $L(M)$ en caso contrario.

13 Para corroborar estas afirmaciones se debe calcular dZ/dPz de D.1 y D.2.2.
} 
Estas conclusiones son compatibles con la condición B.2 del modelo general que implica que en el óptimo, la utilidad marginal del estatus sobre la utilidad marginal de la funcionalidad visible debe subir ante aumentos en el precio del bien $Z$.

Por último, resulta interesante interpretar la demanda del bien $X$ en el óptimo. La función Cobb-Douglas tiene la particularidad de que el coeficiente asociado equivale al porcentaje del gasto total asociado a ese bien. En el caso del bien X, se observa que el gasto total en el mismo equivale a un porcentaje "a\%" del ingreso M más el valor de mercado del estatus autónomo que provee ese ingreso. En otras palabras, la riqueza del individuo radica tanto en su poder de compra como en el estatus que ese poder le representa.

Trabajando con las ecuaciones D.1 y D.2.2 se puede probar que el porcentaje de riqueza dedicado al gasto en la funcionalidad $F_{2}$ valuado únicamente al precio Py (i.e., $\mathrm{Py}{ }^{\star}(\mathrm{Y}+\mathrm{Z})$ ) equivale a un porcentaje "b\%" de la misma dotación. Esto implica que el agente dedica al consumo de estatus (implícita o explícitamente) un porcentaje 1-a-b de la su riqueza (la cual incluye su riqueza intangible en términos de estatus) en términos reales del precio del estatus, el cual es (Pz-Py). ${ }^{14}$

Hasta aquí se han desarrollado las condiciones que implican soluciones interiores al modelo. Asimismo se demostró que la solución de esquina $X=0, Y=0$ no es factible. A continuación se trabajará con la determinación de soluciones de esquina para las variables $Z$ e $Y$.

Si no se cumple la condición E.1, Z adopta valor nulo. En este caso, la solución del problema es la habitual para un modelo Cobb-Douglas de 2 factores. En base a las condiciones C.1 y C.3 se obtienen:

$$
X=\frac{a}{a+b} M \quad \text { (F.1) } \quad \text { y } \quad Y=\frac{b}{a+b} M
$$

Cuando no se cumple la condición E.2, Y adopta valor nulo. Sin embargo la solución no es tan sencilla como en el caso anterior. Resolviendo el problema de maximización y asumiendo que $Y=0$, se obtienen CPOs análogas a B.4. De ahí es posible calcular las demandas de $\mathrm{X}$ y $Z$, a saber:

$$
\begin{aligned}
& Z=\frac{(a+b) P_{Z} L(M)-(1-a) M+\sqrt{\left[(a+b) P_{Z} L(M)-(1-a) M\right]^{2}+4 b M L(M) P_{Z}}}{-2 P_{Z}} \\
& X=a \frac{P_{Z}}{P X} \frac{Z(L(M)+Z)}{(1-a) Z+b L(M)}
\end{aligned}
$$

Nótese que el módulo de la raíz de G.1 es mayor al del resto del numerador, lo cual implica que la ecuación cuadrática que define $Z$ posee una raíz positiva y otra negativa. Dado que la raíz negativa debe ser descartada, esto nos da una solución única. La derivada con respecto a $\mathrm{M}$ de ambas funciones depende de los valores asumidos de los parámetros, pero una vez fijos, de la forma funcional de $L(M)$.

14 Se puede probar que en el óptimo el gasto total es igual a $\mathrm{M}$, con el siguiente cómputo. Gasto Total $=P x^{\star} X+P^{\star}(Y+Z)+(P Z-P y) \star Z$. 


\section{C.3 Racionalización de la curva de Engel en forma de S}

Del modelo desarrollado en la sección precedente se concluye que la existencia de un componente de estatus vinculado a la dotación de los agentes puede afectar sustancialmente la necesidad de recurrir a un mercado implícito de estatus. Más aún, si dicho componente no guarda relación lineal con el monto de la dotación, se da lugar a distintas concavidades ( $\mathrm{y}$ hasta distintos signos) en la curva de Engel de los bienes. En particular, si la función $\mathrm{L}(\mathrm{M})$ posee rendimientos a escala crecientes en un primer momento y decrecientes a partir de cierto umbral de ingreso, es posible construir curvas de Engel en forma de "S" (con pendiente positiva-negativa-positiva). ${ }^{15}$

Si bien este es un fenómeno matemático, es posible racionalizarlo en términos económicos y sociales. $L(M)$ se interpreta como el impacto que tiene la dotación inicial en la percepción de status del agente. Tal como se mencionó dicha dotación es inobservable, pero pasible de ser inferida en base al patrón de consumo general del agente. ${ }^{16}$ Estas inferencias pueden ser explícitas (i.e., se observa al agente consumir) o implícitas (i.e., el consumo de ciertos bienes es inferido por el comportamiento o la apariencia del agente).

Supongamos que un agente cuenta con una dotación de ingresos muy acotada. Los consumos que realice, si bien fundamentales para la supervivencia, pueden no tener mayor impacto en el estatus social, ya que son asumidos como básicos para el hombre. Sin embargo, estos son considerados más como consumos de supervivencia que como consumos de socialización.

Si subimos unos peldaños en el nivel de ingreso, el agente va cubriendo necesidades menos vitales, aunque primordiales y con impacto en su apariencia social: vestimenta, educación, cierto nivel de esparcimiento/bienes culturales, información, transporte. Estas cualidades son básicas para la socialización del agente, por lo que sin ellas no hay posibilidad de estatus social alguno. Son las que permiten tener un nivel de desenvolvimiento social mínimo. Por lo tanto uno puede pensar en un retorno creciente de la dotación inicial sobre la percepción de estatus para los primeros tramos de la curva.

Al escalar aún más en el nivel de riqueza, comienzan a aparecer consumos no tan necesarios y con menor impacto sobre la socialización del agente. Es aquí donde el consumo tradicional (de bienes no suntuarios) adicionales comienza a carecer de sus retornos crecientes sobre la percepción de status.

En un ejemplo concreto, el paso de no tener vestimenta, a tener una camisa harapienta, si bien representa un incremento marginal superior en el consumo de vestimenta, es probable que poco haga por el estatus del agente. El paso de esta camisa a una camisa blanca tradicional probablemente sea un incremento marginal menor en términos de la necesidad de vestimenta, pero uno superior a nivel de estatus. El paso de una camisa blanca tradicional a otra blanca de algodón suizo, es de esperar sea un incremento marginal menor en ambos sentidos. ${ }^{17}$

\footnotetext{
${ }^{15}$ La teoría planteada guarda ciertas similitudes con la teoría de utilidad del dinero de Friedman-Savage (1948), según la cuál la utilidad de los agentes con respecto a M posee una sucesión de tramos cóncavo-convexocóncavo. Según los autores, los tramos con rendimientos crecientes corresponderían al pasaje de estrato social, mientras que los decrecientes a movimientos dentro de un mismo estrato. Notar que en cierta medida, los autores postulan que desde la óptica de un agente particular, existen 2 clases: La suya y la superior; por lo que $\mathrm{U}(\mathrm{M})$ posee solamente 3 tramos (i.e., 2 convexos interrumpidos por uno cóncavo).

16 Esta es otra diferencia con el modelo de Ireland (1994) donde la única inferencia de consumo se da por el nivel de consumo del bien visible.

17 Adam Smith (1776, Libro V, Cap. 2, Art. IV) ya decía que los "Griegos y Romanos pudieron sobrevivir sin camisas de lino, pero que en los tiempos actuales en la mayor parte de Europa sería vergonzoso para un trabajador respetable no utilizar una, ya que denotaría un nivel de pobreza solo esperable en personas de mala conducta."
} 


\section{Primeros Pasos a la Evaluación Empírica}

En esta sección se presenta una primera aproximación empírica de la hipótesis planteada basada en datos de la Encuesta Nacional de Gastos (ENGH 2004/2005) para 10 provincias. ${ }^{18}$ Dicha encuesta fue realizada por el INDEC y provee información detallada de consumo, características demográficas, ingresos y situación laboral para el total del país.

La primera problemática es la elección de los (grupos de) bienes candidatos a comportarse de la forma prevista por la teoría. ${ }^{19}$ Es opinión del autor que los mismos deben reunir 3 requerimientos: a) existencia de un menú de sustitutos en un rango amplio de precios, b) tener un bajo costo relativo y c) su alta visibilidad. Dos grupos de bienes que reúnen estas características son los teléfonos celulares y las zapatillas deportivas.

En ambos casos existen sustitutos casi-perfectos en un rango de precios llamativamente amplio. En el caso de los celulares, hoy existen en el mercado aparatos con precios entre los AR\$ 100 y los AR\$2.000. Todos comparten la misma tecnología de comunicación (GSM), su calidad de sonido es idéntica, todos cuentan con agenda y otras funciones básicas de telefonía, acceso a Internet y su peso, tamaño, y autonomía es similar. Sin embargo difieren sustancialmente en diseño y algunas aplicaciones extra-telefónicas: Cámaras fotográficas, video, reproducción de mp3.

En el caso de las zapatillas deportivas, la sustitución casi-perfecta es más sencilla de argumentar. Para el común de las personas (no deportistas profesionales) la diferencia entre una zapatilla con suela de espuma, una con cámara de aire u otra con resortes es mínima. Esto se debe a que el 95\% del tiempo el calzado es utilizado en la vida diaria de las personas, situación en la que la suela de goma espuma es más que eficiente. Por lo tanto, el consumo de zapatillas con sofisticadas suelas puede asociarse al consumo de bienes de ostentación. Esto lleva a pensar que los productos con precios superiores tienden a asemejarse a los bienes " $Z$ " del modelo antes desarrollado, mientras que los más económicos se relacionan con los bienes "Y".

En cuanto al bajo costo relativo, tanto las zapatillas más sofisticadas cómo la mayoría de los teléfonos celulares más modernos son asequibles con cerca de AR $\$ 600$ y AR $\$ 1.000$ respectivamente. Si bien estas son cifras no despreciables, las mismas representan un nivel de ahorro alcanzable por la mayoría de los sectores de la sociedad (aún los agentes en situación de pobreza). Por el contrario, otros bienes de ostentación (automóviles, joyas, etc.) son imposibles de adquirir por los sectores de ingresos bajos.

Por ultimo, de existir, son pocos los productos personales que tengan mayor exposición en la vida diaria que el teléfono celular, el cuál es transportado (y mostrado) constantemente por las personas. En el caso de las zapatillas, si bien su exposición es más limitada, no deja de ser alta. Es real que hay situaciones en que son inadecuadas, o que las mismas deben ser lavadas, pero esto sucede en mucha menor medida que con el resto de la vestimenta. Más aún, dado que la mayoría de las personas poseen menos pares de calzado que de otras prendas de vestir, es comúnmente aceptado "repetir" el uso del calzado, algo que es muchas veces socialmente condenado en el caso de otras prendas.

\footnotetext{
${ }^{18}$ La elección de la base de datos no fue trivial dados los requerimientos de información de consumo. Se remarca que el autor no contó con acceso directo a dicha base ya que la misma no fue distribuida públicamente. Por lo tanto, el trabajo se realizo a distancia mediante la elaboración de archivos ".do" de Stata los cuales fueron ejecutados por el tutor. Se intento buscar bases de datos alternativas de consultoras privadas pero las mismas no fueron provistas al autor.

${ }^{19}$ En un trabajo relacionado, Heffetz (2007a) realizó una encuesta puntual sobre el efecto del consumo de 29 categorías de bienes sobre la percepción de estatus, lo cual fue utilizado para construir un índice de visibilidad de bienes para Estados Unidos. Si bien el índice de visibilidad es un aporte sustancial en virtud del presente análisis, su especificidad no es suficiente para evaluar la hipótesis planteada. Sin embargo, se recalca que en dicho trabajo los celulares y la vestimenta son considerados grupos relevantes como proveedores de estatus.
} 
Como conclusión, su alta visibilidad, su bajo costo relativo y la existencia de sustitutos casiperfectos postula a los teléfonos celulares y a las zapatillas como bienes candidatos a presentar los comportamientos de "S" en su curva de Engel, tal como fue postulado en la parte teórica precedente.

En particular sobre el consumo de teléfonos celulares, últimamente han aparecido diversas noticias en los medios referidos validando la hipótesis desarrollada. Según lo publicado en el periódico Infobae el 22 de agosto de 2008, la consultora LatinPanel realizó un estudio con 9.500 observaciones concluyendo que "la sofisticación del equipo (de telefonía celular) cobra mayor importancia a medida que decrece el nivel socioeconómico de los usuarios." Desde ya, esto puede deberse a un fenómeno de estatus (tal como el aquí analizado) o a que el teléfono celular es utilizado como sustituto de otros bienes (computadora, video juego, etc.) por parte de los sectores bajos de la sociedad. ${ }^{20}$

La ENGH 2005 cuenta con información de consumo de los hogares abierta en 1.156 categorías, dentro del último año previo a la encuesta. ${ }^{21}$ La base de datos obtenida es parcial, y cuenta con observaciones para 10 provincias. $^{22}$ La misma contiene 11.701 observaciones (hogares), de las cuales 120 reportan algún componente de gasto total negativo. En una primera instancia se consideró que esto implicaba venta de existencias, pero el consumo negativo no coincide con lo reportado en las ventas de productos por parte de las familias (variable gasvent); razón por la cuál se eliminaron dichas observaciones.

De interés para el presente estudio son los siguientes artículos descriptos en la ENGH: 622102 - Equipos móviles de teléfono; 221107 - Zapatillas o calzado deportivo para hombres y 221207 -Zapatillas o calzado deportivo para mujeres. Para cada artículo, se informa monto y cantidad unitaria de bienes consumidos, permitiendo obtener en la mayoría de los casos el precio promedio pagado por cada familia en cada uno de los bienes. Respectivamente, 80, 1.111, y 826 hogares reportan consumo de cada uno de los bienes analizados.

Se remarca que para algunas observaciones solamente se provee la variable monto pero no la variable cantidad (12 casos para teléfonos celulares, 298 para calzado masculino y 205 para femenino). En esos casos, se opto por trabajar con dos muestras: uno que ignore dichas observaciones, y otro que estime el componente faltante. La razón de este modelo dual es que se temía que dado que el objeto del análisis es explicar la variación de precios, la selección de variables explicativas a utilizar para completar la muestra podría distorsionar el análisis final. En efecto ese fue el resultado: las regresiones relacionadas con el calzado ${ }^{23}$ brindan resultados similares con ambas muestras, pero significativamente más robustos para la muestra extendida.

${ }^{20}$ El 24 de Abril de 2009, un nota publicada en el diario El Cronista reporta que un relevamiento de 1.000 hogares del segmento bajo y medio-bajo estima que un porcentaje importante de usuarios utilizan al celular como un medio de entretenimiento (frente a la PC). Sin embargo, también se destaca que "el uso del móvil gira en torno a las funcionalidades más básicas: mensajes y llamadas." Otro artículo publicado en el Diario Clarín del día 22 de Febrero de 2009 argumenta que el alto gasto de los sectores cadenciados en telefonía celular se da no por prestigio sino por la posibilidad de comunicación y la seguridad que brinda. Se remarca que este último artículo se refiere al consumo de servicios telefónicos y no a la compra de equipos.

${ }^{21}$ Se remarca que la ENGH no es precisa al detallar el periodo de referencia del consumo de bienes durables, al menos en la información disponible. Para los bienes durables el periodo de referencia varia entre 1 mes y 1 año.

${ }^{22}$ Capital Federal, Salta, Jujuy, Tucumán, Chaco, Santiago del Estero, Mendoza, La Rioja, Misiones y Río Negro.

${ }^{23}$ Para estimar las cantidades de calzado faltante se terminó utilizando una regresión binomial negativa de las cantidades positivas consumidas frente a una única variable explicativa (el monto gastado). Se remarca que tanto para calzado femenino como masculino, se encontraron dos observaciones donde había declarada una cantidad positiva de consumo pero ningún monto. En este caso se estimo por MCO regresando todos los montos positivos en cantidades, ingreso por adulto equivalente, porcentaje del gasto total en indumentaria y calzado por parte del hogar, y el nivel de instrucción del jefe de hogar. Todos los coeficientes fueron positivos y significativos. 
La Tabla 1 muestra los precios de las zapatillas, montos y cantidades por cada decil de ingreso equivalente. Se observa que los deciles poseen una relación casi lineal entre la posición 2 y 8 , creciendo exponencialmente en los extremos. Sin embargo, el porcentaje de ingreso gastado por cada grupo en el rubro indumentaria y calzado es relativamente estable en torno al $8.2 \%$.

Una primera observación es que la cantidad de hogares que reportan consumo de calzado deportivo disminuye abruptamente en los deciles superiores, mientras que se mantiene estable en los medios/bajos. Este factor puede interpretarse tanto dentro de la teoría planteada (los sectores bajos invierten más en calzado debido a su componente de imagen) como desde la teoría de uso del tiempo (el valor tiempo del dinero de los sectores altos los desvía de actividades de ocio tiempo-intensivas como ser el deporte).

En cuanto a los precios de consumo, se observa una correlación positiva entre ingreso y precio del calzado masculino, pero con un marcado amesetamiento entre los deciles 5 y 7 , lo cual puede ser interpretado como evidencia (parcial) de la teoría aquí planteada. Para calzado femenino, si bien no tan marcado, se observa un abrupto cambio de pendiente en el decil 5, contrarrestado por notorias bajas en los precios de consumo en los deciles 7 y 9.

\section{Análisis Descriptivo: Calzado Deportivo}

\begin{tabular}{|c|c|c|c|c|c|c|c|c|c|c|}
\hline \multirow[b]{2}{*}{ Decil AEQ } & \multirow[b]{2}{*}{$\begin{array}{l}\text { Ingreso } \\
\text { medio }\end{array}$} & \multirow[b]{2}{*}{$\begin{array}{l}\text { Porcentaje de Gasto en } \\
\text { Indumentaria y Calzado }\end{array}$} & \multicolumn{4}{|c|}{ Zapatillas Masculinas } & \multicolumn{4}{|c|}{ Zapatillas Femeninas } \\
\hline & & & Precio Observado & Precio Estimado & Monto & $\begin{array}{c}\text { Cantidad de } \\
\text { Hogares } \\
\text { Consumidores }\end{array}$ & $\begin{array}{c}\text { Precio } \\
\text { Observado }\end{array}$ & $\begin{array}{l}\text { Precio } \\
\text { Estimado }\end{array}$ & Monto & $\begin{array}{c}\text { Cantidad de } \\
\text { Hogares } \\
\text { Consumidores }\end{array}$ \\
\hline \multicolumn{11}{|c|}{ Observaciones } \\
\hline 1 & 73 & $7.8 \%$ & 35.1 & 39.8 & 43.4 & 114 & 27.7 & 29.1 & 36.7 & 83 \\
\hline 2 & 132 & $8.8 \%$ & 43.3 & 43.8 & 51.7 & 141 & 33.4 & 35.7 & 39.2 & 107 \\
\hline 3 & 183 & $9.5 \%$ & 47.4 & 54.0 & 59.4 & 147 & 39.0 & 44.8 & 51.8 & 129 \\
\hline 4 & 239 & $8.9 \%$ & 59.1 & 58.2 & 61.8 & 142 & 41.0 & 43.6 & 48.9 & 126 \\
\hline 5 & 310 & $8.8 \%$ & 73.4 & 73.8 & 82.7 & 141 & 53.9 & 54.4 & 60.9 & 95 \\
\hline 6 & 406 & $8.0 \%$ & 69.9 & 70.8 & 84.0 & 128 & 62.2 & 62.0 & 63.8 & 76 \\
\hline 7 & 532 & $7.2 \%$ & 72.8 & 74.5 & 89.3 & 113 & 44.5 & 50.4 & 55.3 & 70 \\
\hline 8 & 725 & $8.1 \%$ & 87.0 & 85.7 & 93.2 & 91 & 72.8 & 69.3 & 74.5 & 68 \\
\hline 9 & 1,087 & $7.1 \%$ & 104.6 & 102.3 & 103.6 & 56 & 67.1 & 69.3 & 71.5 & 51 \\
\hline 10 & 2,558 & $7.6 \%$ & 122.6 & 120.0 & 120.0 & 37 & 81.0 & 75.7 & 76.9 & 21 \\
\hline
\end{tabular}

En el caso de los teléfonos celulares, se encontró que de los 68 hogares que declaraban consumo ninguno superaba la unidad, pero una gran cantidad declaraban consumo de una fracción de bien. Descartada la opción de compra a crédito (la cual debería capturarse por la variable gasto a crédito), se puede asumir que estos agentes comparten un equipo celular entre varios hogares, que se adquieren equipos en comodato o que se trata de un error en la base. La alternativa en este caso fue asumir que todos los hogares que reportan gasto en el bien adquirieron una unidad, por lo que el precio equivale al monto reportado. Por ultimo, como estadística descriptiva, se reportan los $X$ casos en los que se reportan efectivamente una unidad de consumo

La tabla 2 muestra el análisis descriptivo para la compra de equipos de telefonía celular. Asimismo, se agregan dos columnas que intentan captar la intensidad de uso de los teléfonos celulares, al dividir el gasto en el equipo sobre el consumo en servicios de telefonía móvil. ${ }^{24}$ Los resultados, si bien muestran cierta tendencia, deben analizarse teniendo en cuenta el tamaño pequeño de la muestra.

En primer lugar, a excepción de los primeros dos deciles, aquí se nota que la cantidad de hogares consumidores es relativamente estable. Tanto en precio como en monto, la calidad del bien consumido por los deciles extremos es similar, sin embargo el decil 9 muestra el mayor precio/monto de consumo. A nivel monto, sin embargo, es relevante observar que los deciles 3, 6, 7 y 8 muestran patrones de consumo similares.

Por último, en cuanto a la intensidad de uso de los equipos celulares, se observa que los deciles 4 y 6 son los que menos aprovechan las funcionalidades pagas de los equipos

\footnotetext{
${ }^{24}$ Para el caso del calzado deportivo no se incluyo una medida similar ya que es difícil medir el uso que se le da a las zapatillas deportivas. Desde ya el consumo de cuotas en clubs o alquileres de canchas podría considerarse como un proxy, aunque el mismo estaría altamente sesgado por la omisión de practicas deportivas en espacios públicos o propios.
} 
celulares. Al respecto, cabe aclarar que es posible que los sectores bajos utilicen el equipo de telefonía celular como centro de entretenimiento (i.e., videojuegos, mp3) mientras que los sectores altos tengan bienes alternativos para esos usos; lo cual puede estar distorsionando la medida de usufructo del bien.

Análisis Descriptivo: Equipos de Telefonía Celular

\begin{tabular}{|c|c|c|c|c|c|c|c|}
\hline Decil AEQ & $\begin{array}{c}\text { Ingreso } \\
\text { Medio }\end{array}$ & $\begin{array}{c}\text { Porcentaje de Gasto en } \\
\text { Comunicaciones }\end{array}$ & $\begin{array}{c}\text { Precio Observado } \\
\text { Observaciones }\end{array}$ & $\begin{array}{c}\text { Monto } \\
\text { Cantidad de } \\
\text { Hogares } \\
\text { Consumidores }\end{array}$ & Precio/Gasto* & Monto/Gasto* \\
\hline $\mathbf{1}$ & 73 & $5.9 \%$ & 164.5 & 79.8 & 3 & 2.7 & 1.3 \\
$\mathbf{2}$ & 132 & $7.9 \%$ & 106.0 & 53.0 & 3 & & \\
$\mathbf{3}$ & 183 & $8.7 \%$ & 192.3 & 92.4 & 8 & 8.0 & 5.2 \\
$\mathbf{4}$ & 239 & $10.1 \%$ & 121.2 & 65.4 & 8 & 13.5 & 8.6 \\
$\mathbf{5}$ & 310 & $11.3 \%$ & 139.9 & 65.9 & 13 & 3.7 & 3.0 \\
$\mathbf{6}$ & 406 & $13.1 \%$ & 217.5 & 92.4 & 7 & 15.6 & 7.9 \\
$\mathbf{7}$ & 532 & $14.3 \%$ & 178.5 & 98.2 & 8 & 3.9 & 3.5 \\
$\mathbf{8}$ & 725 & $14.6 \%$ & 148.3 & 91.0 & 11 & 5.2 & 4.5 \\
$\mathbf{9}$ & 1,087 & $15.9 \%$ & 257.0 & 108.5 & 9 & 9.5 & 5.3 \\
$\mathbf{1 0}$ & 2,558 & $15.6 \%$ & 169.2 & 77.7 & 10 & 6.4 & 4.3 \\
\hline
\end{tabular}

* Solo para hogares que reportan Adquisicion de Telefonos Celulares

El siguiente gráfico concluye el análisis descriptivo. En el mismo se muestra el ingreso medio por hogar por grupos de consumo de cada uno de los bienes analizados. Los grupos de consumo son definidos por los deciles de precio de los bienes analizados. Si los bienes se comportasen normalmente, el ingreso medio de los grupos de consumo debería ser similar al del ingreso por adulto equivalente en cada decil. Notar que el ingreso crece de decil en decil en forma exponencial, mientras que el ingreso equivalente de los deciles de precio de los bienes analizados siguen un patrón irregular (principalmente en el caso de los celulares lo cual puede estar afectado por el número de observaciones) y con mínimo o nulo incremento hasta el decil 6/7. Si se remarca que el ingreso promedio de los consumidores de los bienes más caros es significativamente superior al resto.

Ingreso Medio por Adulto Equivalente de los Hogares Consumidores

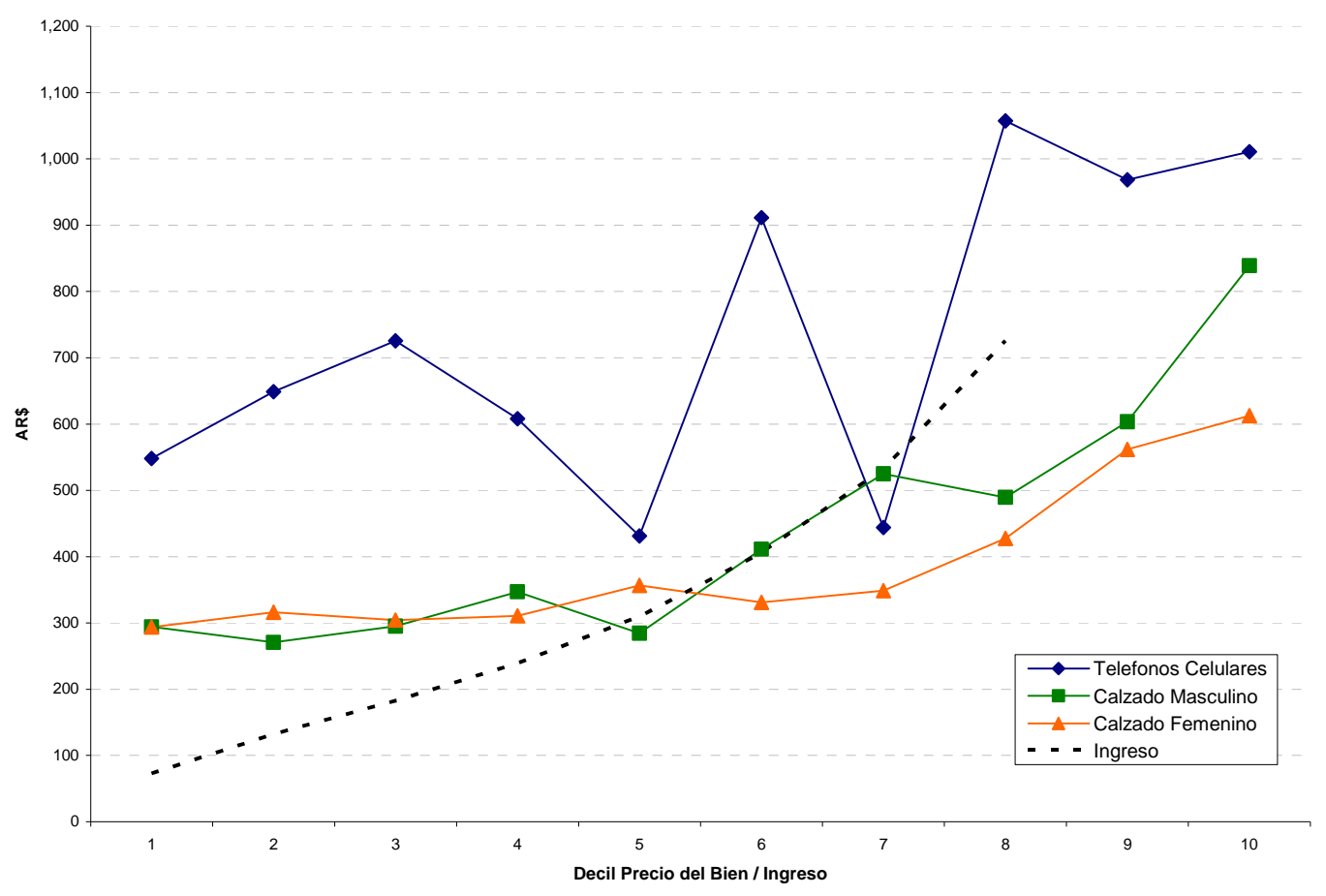


A continuación, se trabaja con el análisis condicional. El modelo a estimar es un modelo de datos censurados en el que solamente una parte del los hogares reporta un consumo positivo de los bienes analizados.

Es decir, que:

$$
\begin{aligned}
& \text { Precio/Monto del Consumo }=F(X)+\varepsilon_{i} ; \text { y } \\
& \text { Decisión de Consumo }=F(Y)+\gamma_{i}
\end{aligned}
$$

Si la decisión de cuanto pagar por el consumo (a) fuese independiente de la decisión de consumo (b), (a) podría estimarse consistentemente por MCO. ${ }^{25}$

Sin embargo, dado que es probable que ambas decisiones se encuentren interrelacionadas. En este caso la esperanza de $\varepsilon_{\mathrm{i}}$ no es 0 , sino que esta condicionada por la decisión de consumo positiva. Heckman (1979) propuso un modelo en dos etapas en la que primero se calculo un modelo de tipo probit para la decisión de consumo b, y utilizando la inversa del ratio de Mills (i.e., el valor de la función de densidad sobre la función de probabilidad acumulada) la estimada para cada agente como una variable explicativa adicional a la regresión de (a) por MCO. Esto permite estimar en forma simultánea la decisión de consumo positivo y luego la "calidad" del bien adquirido.

A continuación se presentan las regresiones tipo Heckman realizadas tanto para montos como para precios para cada uno de los bienes, ${ }^{26}$ en busca de verificar si la relación entre precio/monto gastado en estos bienes sigue un patrón no homogéneo con respecto al ingreso. Los precios y montos consumidos son regresados frente al logaritmo del ingreso por adulto equivalente del hogar hasta la tercer potencia (para permitir respuestas no homogéneas ante cambios en el ingreso), la cantidad de bienes adquiridos, el nivel de instrucción del jefe de hogar, el sexo y la edad del jefe de hogar. En el caso de los celulares se agrega una variable dummy que adopta valor si el hogar cuenta con un teléfono de línea.

En la ecuación de selección se utilizan las mismas variables (aunque el ingreso equivalente elevado a la tercera potencia no se incluye para el calzado femenino y para el consumo de celulares) y se suma del gasto total dedicado por el hogar a la categoría amplia del bien analizado (comunicaciones para el caso del celular e indumentaria y calzado para el caso de las zapatillas deportivas).

Se nota que las regresiones vinculadas al consumo de teléfonos celulares poseen un bajo nivel de significatividad agregada (lo cual fue consistente a varias especificaciones probadas) y en principio podría originarse en el reducido tamaño de la muestra. Sin embargo la muestra de montos (más extensa) muestra una menor significatividad que la muestra de precios. Por lo tanto el análisis de resultados se basa exclusivamente en la regresión de precios.

\footnotetext{
${ }^{25}$ Inicialmente se habían calculado también regresiones por MCO incluyendo únicamente observaciones con consumos positivos. Se remarca que MCO continua siendo consistente si se supone que la variable de estudio (en este caso el precio o el monto) es independiente de los factores que provocan un consumo positivo. Este puede ser el caso de estos bienes, ya que por ejemplo una persona de bajos recursos que va a utilizar poco el celular puede decidir comprar un celular si y solo si compra un celular de "estatus". Otras razones pueden ser que temporalmente los celulares fueron ofertados con posterioridad a los sectores bajos de la población (debido al la oferta creciente de equipos sofisticados sin modalidad contractual).

${ }^{26}$ Se remarca que se probaron especificaciones alternativas a las presentadas, las cuales no se presentan dado que los resultados no presentan diferencias significativas.
} 


\begin{tabular}{|c|c|c|c|c|c|c|}
\hline \multirow{2}{*}{ Heckman } & \multicolumn{2}{|c|}{ Zapatillas Femeninas } & \multicolumn{2}{|c|}{ Zapatillas Masculinas } & \multicolumn{2}{|c|}{ Tel'efonos Celulares } \\
\hline & Precio Estimado & Monto Observado & Precio Estimado & Monto Observado & Precio Observado & Monto Observado \\
\hline \multicolumn{7}{|l|}{ Ecuación Censurada } \\
\hline \multirow[t]{2}{*}{ LN(INGAEQ) } & -246 & -325 & 60 & -3 & $-1,468$ & $-1,171$ \\
\hline & $6 \%$ & $3 \%$ & $52 \%$ & $98 \%$ & $38 \%$ & $22 \%$ \\
\hline \multirow{2}{*}{ LN(INGAEQ)^2 } & 44.1 & 57.2 & -12.3 & -0.1 & 234.8 & 191.0 \\
\hline & $6 \%$ & $3 \%$ & $43 \%$ & $100 \%$ & $39 \%$ & $22 \%$ \\
\hline \multirow[t]{2}{*}{ LN(INGAEQ)^3 } & -2.3 & -3.0 & 1.1 & 0.4 & -12.6 & -10.3 \\
\hline & $9 \%$ & $5 \%$ & $21 \%$ & $75 \%$ & $39 \%$ & $22 \%$ \\
\hline \multirow[t]{2}{*}{ Nivel Instrucción Jefe } & - & - & - & - & 16.3 & 5.4 \\
\hline & & & & & $5 \%$ & $20 \%$ \\
\hline \multirow[t]{2}{*}{ Edad Jefe } & -0.5 & -0.6 & -0.2 & -0.2 & 2.8 & 1.3 \\
\hline & $0 \%$ & $0 \%$ & $2 \%$ & $6 \%$ & $2 \%$ & $8 \%$ \\
\hline \multirow[t]{2}{*}{ Sexo Jefe } & -0.2 & 0.1 & 8.7 & 10.3 & 31.3 & 12.1 \\
\hline & $95 \%$ & $98 \%$ & $2 \%$ & $2 \%$ & $23 \%$ & $43 \%$ \\
\hline \multirow[t]{2}{*}{ Hogar con Teléfono Fijo } & 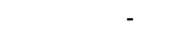 & 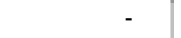 & - & & 14.1 & 13.6 \\
\hline & & & & & $78 \%$ & $61 \%$ \\
\hline \multirow[t]{2}{*}{ Constante } & 549.2 & 726.9 & -26.7 & 97.7 & $3,356.9$ & $2,589.1$ \\
\hline & $2 \%$ & $1 \%$ & $88 \%$ & $67 \%$ & $32 \%$ & $18 \%$ \\
\hline \multicolumn{7}{|l|}{ Ecuación de Selección } \\
\hline \multirow[t]{2}{*}{ LN(INGAEQ) } & \multirow{2}{*}{\multicolumn{2}{|c|}{$\begin{array}{c}1.24 \\
0 \%\end{array}$}} & \multicolumn{2}{|c|}{2.63} & 1.30 & 1.24 \\
\hline & & & & $\%$ & $3 \%$ & $2 \%$ \\
\hline \multirow{2}{*}{ LN(INGAEQ)^2 } & \multirow{2}{*}{\multicolumn{2}{|c|}{$\begin{array}{c}-0.12 \\
0 \%\end{array}$}} & \multicolumn{2}{|c|}{$\begin{array}{l}2 \% \\
-\mathbf{0 . 3 8}\end{array}$} & -0.09 & -0.09 \\
\hline & & & \multicolumn{2}{|c|}{$5 \%$} & $6 \%$ & $4 \%$ \\
\hline \multirow[t]{2}{*}{ LN(INGAEQ)^3 } & \multirow{2}{*}{\multicolumn{2}{|c|}{-}} & & 0 & - & - \\
\hline & & & 13 & $\%$ & - & - \\
\hline \multirow[t]{2}{*}{ Nivel Instrucción Jefe } & \multirow{2}{*}{\multicolumn{2}{|c|}{-0.03}} & & - & -0.07 & -0.04 \\
\hline & & & & & $1 \%$ & $6 \%$ \\
\hline Edad Jefe & $0 .($ & 00 & & 00 & -0.01 & -0.01 \\
\hline & 10 & $\%$ & 21 & $\%$ & $0 \%$ & $0 \%$ \\
\hline Sexo Jefe & $0 .($ & 03 & & 32 & 0.02 & 0.03 \\
\hline & 41 & $\%$ & & $\%$ & $84 \%$ & $77 \%$ \\
\hline Gasto en Comunicaciones & & - & & - & 1.60 & 1.52 \\
\hline & & & & & $0 \%$ & $0 \%$ \\
\hline Gasto Indumentaria/Calzado & 3. & 99 & & 08 & - & - \\
\hline & 09 & $\%$ & & $\%$ & - & - \\
\hline Constante & -5 & .1 & & .1 & -6.5 & -6.0 \\
\hline & 09 & $\%$ & 0 & $\%$ & $0 \%$ & $0 \%$ \\
\hline Lambda & -42.31 & -54.60 & $\begin{array}{c}\mathbf{- 3 6 . 8 9} \\
0.0 \%\end{array}$ & -47.91 & $\begin{array}{c}-133.39 \\
4.2 \%\end{array}$ & $\begin{array}{l}-87.34 \\
3.5 \%\end{array}$ \\
\hline Obs. Selección & 10,8 & 837 & & 553 & 11,500 & 11,485 \\
\hline Obs. Censuradas & 82 & 25 & & 109 & 62 & 77 \\
\hline Wald Chi2 & $0 \%$ & $0 \%$ & $0 \%$ & $0 \%$ & $7.9 \%$ & $29 \%$ \\
\hline
\end{tabular}

En Negrita se marcan los coeficientes significativos a menos del 10\%. La segunda línea muestra el nivel de significatividad de los coeficientes. Las variables utilizadas en cada una de las regresiones difieren por motivos ajenos al autor referidos al acceso a la base de datos.

La principal variable de interés del estudio (el ingreso por adulto equivalente) resulta no significativa en la regresión objetivo para calzado masculino y para teléfonos celulares. Si bien estos no son los resultados esperados por la teoría desarrollada, tampoco son resultados derivados de la teoría convencional que los asocia con bienes (al menos) normales. Es decir, se concluye que el ingreso por adulto equivalente no es una variable relevante para explicar variaciones en el precio de consumo de los bienes analizados. Para el caso del calzado femenino, por el contrario, los coeficientes son significativos y con signo alternado. Sin embargo, si bien la curva de Engel resultante adopta una forma de $\mathrm{S}$, el precio/monto mínimo predicho se da a niveles de ingreso cercanos a la media del primer decil. Por lo tanto, puede concluirse que dentro del rango relevante la demanda de calzado femenino se comporta de acuerdo a lo predicho en la teoría tradicional.

En cuanto al rol del ingreso en la ecuación de selección, aquí el mismo es significativo en todos los casos, y con signo alternado entre potencias. Sin embargo el impacto sobre el índice es distinto en cada uno de los casos en el rango relevante de ingresos (hasta AR\$ 2.200). Para el calzado masculino, el índice probit decrece vis-a-vis el incremento del ingreso, aunque a tasa decreciente. Para los equipos de telefonía celular, por el contrario, la relación es siempre positiva aunque fuertemente decreciente, resultando en un índice estabilizado a partir del nivel de AR $\$ 500$ de ingreso por adulto equivalente. Para el calzado 
femenino, la probabilidad de consumo tiene relación positiva con el ingreso hasta los AR\$ 200 y luego negativa (y prácticamente lineal en el índice).

El nivel de instrucción del jefe de hogar posee signo positivo en la regresión del precio del teléfono celular. La expectativa del autor era encontrar el signo opuesto (una vez controlado por el ingreso) dado que se presuponía que un mayor nivel educativo elevaba el nivel de estatus autónomo de los agentes y por ende la necesidad de buscar estatus en el mercado se reducía. Sin embargo, también es posible que un mayor nivel educativo se correlacione con un menor costo de manejo de tecnología "sofisticada". Para la ecuación de selección el nivel educativo posee en todos los casos un coeficiente negativo, aunque su magnitud es poco relevante.

La edad del jefe de hogar probó tener un impacto negativo en el monto/precio gastado en calzado y positivo en el precio del equipo de telefonía móvil adquirido; mientras que posee un impacto virtualmente nulo en la probabilidad de consumo. Los hogares con jefe mujer tienden a comprar menos calzado masculino pero a un precio más alto.

En el caso de los equipos celulares, no se evidencia ni complementariedad ni sustitución entre las cualidades de los teléfonos celulares más sofisticados y la posesión de una línea de telefonía fija.

Por último, la probabilidad de consumo aumenta con el share de consumo dedicado por el hogar en la categoría amplia del bien analizado.

Para el caso del precio de las zapatillas masculinas (que resulto el empíricamente más interesante en relación a la teoría planteada) se corrieron regresiones análogas a las anteriores pero para cada provincia y para cada decil de ingreso equivalente.

La regresión por provincia arrojó coeficientes no significativos para las tres potencias del ingreso en 9 de las 10 Provincias. Para la Provincia de Chaco, sin embargo, los coeficientes son altamente significativos, y de signo alternado. Sin embargo, como en el caso del calzado femenino, el mínimo de consumo se da a niveles muy bajos de ingreso por adulto equivalente, pero aquí se observa un máximo en un rango elevado pero factible de ingreso equivalente (AR\$ 1.400). ${ }^{27}$

Al realizar la regresión del monto por decil de ingreso equivalente, se utiliza solamente la primera potencia del ingreso por adulto equivalente, ya que la muestra ya se encuentra seccionada de acuerdo a la escala de ingreso. Aquí se encontró no significatividad de la variable ingreso en todos los deciles salvo en 3: el 2 (con un valor de coeficiente de 56.2), el 3 (90.4) y el 10 (73.4). Es decir que los sectores de bajos recursos deciden invertir una parte sustancial de su aumento en el ingreso en calzado deportivo masculino, mientras que los otros sectores (a excepción del más rico) no reaccionan de igual manera.

\footnotetext{
${ }^{27}$ Los coeficientes obtenidos son $-785.12,142.15$ y -8.07 para la primera, segunda y tercer potencia respectivamente.
} 


\section{Conclusión}

El presente trabajo postula un modelo novedoso sobre el consumo de bienes de estatus por parte de los agentes. A diferencia de trabajos anteriores, aquí no se intenta explicar el consumo de bienes suntuarios por los estratos bajos (lo cuál puede ser estudiado bajo la literatura de clubes y diferenciación dentro de los mismos) sino el hecho de que el patrón de consumo sea no uniformemente creciente a medida que ascendemos en la escala socioeconómica.

Las características distintivas del modelo presentado son la incorporación de un consumo autónomo de estatus por los agentes, el cuál depende de la dotación, y que el bien relacionado con el estatus posee cualidades funcionales no triviales.

La evaluación empírica no produjo resultados contundentes. Por un lado, y en compatibilidad con lo predicho en la teoría no se observan diferencias estadísticas entre el tipo de teléfono celular y calzado deportivo consumido por los estratos sociales extremos. Por el contrario, tampoco se observa una menor calidad de consumo en los sectores medios. El análisis descriptivo, por su parte, corrobora un amesetamiento en la suba de la calidad de los bienes consumidos frente a aumentos en el ingreso por los sectores medios. Por lo tanto es posible que sin llegar a tener tramos de elasticidad ingreso negativas, dichos bienes exhiban una fuerte caída en la elasticidad ingreso en los sectores medios de la sociedad.

La recomendación de política usual frente a la existencia de estos fenómenos es bregar por una mayor carga impositiva sobre los bienes de "estatus", o por el uso de transferencias en especies que eviten el "derroche" de recursos. ${ }^{28}$ Sin embargo, dado que el estatus es un argumento mismo de la función de utilidad, y que existe un componente autónomo del mismo, es opinión del autor que la sociedad generara un mercado alternativo de estatus a medida que el existente se vuelva inviable por su carga fiscal.

Por el contrario, cobra relevancia el rol fundamental - ya enfatizado por Moffit (1983) - que tiene el diseño de políticas sociales que no generen un estigma social: así el rol de programas (mayormente culturales) que eleven el nivel de estatus autónomo de los individuos. De esta forma, la necesidad de buscar estatus en el mercado tendería a disminuir, lo cual implica más recursos dedicados a bienes "funcionales".

\footnotetext{
${ }^{28}$ Siempre desde el punto de vista del planificador social, ya que desde la óptica del agente siempre será mejor poseer el máximo de grados de libertad posible.
} 


\section{Bibliografía}

Basmann R.L., Molina D.J., Slottje D.J. (1988): “A Note on Measuring Veblen's Theory of Conspicuous Consumption". The Review of Economics and Statistics, Vol. 70, No. 3, pp. 531-535.

Becker G.S., Murphy K.M., Werning I. (2003): "The Equilibrium Distribution of Income and the Market for Status." Available at: http://econ-www.mit.edu/files/1270.

Carlsson F., Johansson-Stenman O., Martinsson P., (2003). "Do You Enjoy Having More Than Others? Survey Evidence of Positional Goods," Working Papers in Economics 100, Göteborg University, Department of Economics.

Charles K., Hurst E. Roussanov N. (2007). "Conspicuous Consumption and Race," mimeo, Agosto.

Chao A., Schor J.B. (1996): "Empirical tests of status consumption : evidence from women's cosmetics". WORC Paper number 7, Work and Organization Research Centre, Tilburg University.

Clarin: "El celular de los pobres," 22 de Febrero de 2009.

Clark A.E., Frijters P., Shields M.A., (2007): "Relative Income, Happiness and Utility: An Explanation for the Easterlin Paradox and Other Puzzles". IZA Discussion Paper No. 2840.

Easterlin R. (1995): "Will raising the incomes of all increase the happiness of all?". Journal of Economic Behavior and Organization, Vol. 27 (1), pp. 35-47.

El Cronista: "El celular atrae a los sectores más pobres," 24 de Abril de 2009.

Frank R.H. (1985): "The Demand for Unobservable and Other Nonpositional Goods," American Economic Review, American Economic Association, Vol. 75 (1), pp. 101-16.

Friedman M., Savage L.J. (1948): "The Utility Analysis of Choices Involving Risk". The Journal of Political Economy, Vol. 56 (4), pp. 279-304.

Hayakawa H., Yiannis V. (1977): "Consumer Interdependence via Reference Groups". Journal of Political Economy, Vol. 85, No. 3, pp. 599-615.

Heffetz O. (2007a). "A Test of Conspicuous Consumption: Visibility and Income Elasticities" Cornell University - S.C. Johnson Graduate School of Management. Agosto.

Heffetz O. (2007b). "Cobb-Douglas Utility With Nonlinear Engel Curves in a Conspicuous Consumption Model". Cornell University - S.C. Johnson Graduate School of Management. Septiembre.

Himadri R., Majumdar S. (2006). "Of Diamonds and Desires: Understanding Conspicuous Consumption from a Contemporary Marketing Perspective," Academy of Marketing Science Review, Vol 2006, No 11.

INDEC, Encuesta Nacional de Gasto de los Hogares (2004-2005), Noviembre de 2007.

Infobae: "Menor poder adquisitivo, más interés en los móviles de última generación," 22 de agosto de 2008.

Ireland N. (1994). "On limiting the market for status signals". Journal of Public Economics, Vol. 53 (1), pp. 91-110

Lamont M. and Virag M. (2001), "How Blacks Use Consumption to Shape their Collective Identity," Journal of Consumer Culture 1, no. 1.

Levy S.J. (1959): "Symbols for Sale". Harvard Business Review, Julio-Agosto, pp. 117-124.

Liebenstein H. (1950): "Bandwagon, Snob and Veblen Effects in the theory of consumer's demand". Quarterly Journal of Economics, 64, pp. 183-207.

Luttmer Erzo F.P. (2005): "Neighbors as negatives: relative earnings and well-being". NBER, Working Paper 10667.

McBride M. (2001): "Relative-Income Effects on Subjective Well-Being in the Cross-section". Journal of Economic Behavior and Organization XLV, pp. 251-278.

Moffit R. (1983): “An Economic Model of Welfare Stigma," The American Economic Review, Vol. 73, No. 5, pp. 1023-1035. 
Pérez Truglia R.N. (2007): "Can a rise in income inequality improve welfare?". Anales de la AAEP, 2007.

Rosen S., (1974): "Hedonic Prices and Implicit Markets: Product Differentiation in Pure Competition". Journal of Political Economy, University of Chicago Press, Vol. 82(1), pp. 34-55.

Smith A., (1776): "An Inquiry into the Nature and Causes of the Wealth of Nations."

Stigler G., Becker G. (1977): "De Gustibus Non Est Disputandum," American Economic Review, American Economic Association, Vol. 67 (2), pp. 76-90.

Van de Stadt H., Kapteyn A., Van de Geer S. (1985): "The Relativity of Utility: Evidence from Panel Data". Review of Economics and Statistics, May 1985, 67(2), pp. 179-87.

Veblen T., (1894): "The economic theory of Women's dress". Popular Science Monthly, Vol. XLVI.

Veblen T., (1899): "The Theory of the Leisure Class: an economic study of institutions". New York, London. Macmillan. 